\title{
Streptococcus suis and an Incidentally Diagnosed Metastatic Colon Cancer
}

\author{
Authors: \\ *Maha Osman Mohamed Shangab, Niaz Ahmed Shaikh \\ Rashid Hospital, Dubai Health Authority, Dubai, United Arab Emirates \\ *Correspondence to moshangab@dha.gov.ae \\ Disclosure: \\ The authors have declared no conflicts of interest. \\ Received: \\ 31.05 .20 \\ Accepted: \\ 28.07.20 \\ Keywords: \\ Colon cancer, Streptococcus spp, Streptococcus suis. \\ Citation: \\ EMJ. 2021; DOI/10.33590/emj/20-00140.
}

\section{Abstract}

Background: Streptococcus suis is a zoonotic infection known to cause meningitis and sepsis, in addition to several other rare manifestations. Infection with this organism is rare in the absence of pork ingestion or a handling history.

Case presentation: The authors report the case of a 62-year-old male with no animal contact history, who presented with symptoms of urinary tract infection. It was his second infection over the course of 2 years. His urine culture was positive for Escherichia coli but his blood culture was positive for S. suis. Ultrasound of the abdomen ruled out underlying predisposing urinary pathology. However, it did show several heterogeneous liver masses with abnormal vascularity. A follow-up abdominal CT revealed a malignant neoplastic process involving the sigmoid colon with metastatic liver lesions. Colonoscopy demonstrated a fungating mass at the sigmoid colon and biopsies revealed a moderately differentiated adenocarcinoma.

Conclusion: This case suggests the possibility of associated colon cancer in patients presenting with S. suis with no explicit history of animal or pork contact. It also proposes the existence of an association between colon cancer with Streptococcus species other than bovis.

\section{BACKGROUND}

Streptococcus suis is a zoonotic gram-positive bacterium that is usually acquired through direct contact with swine or pork. In humans, this organism can present as acute meningitis, septicaemia, infective endocarditis, or other manifestations. 'Patients with S. suis meningitis can develop permanent hearing loss. Male sex and occupations with direct swine or pork contact are considered risk factors., ${ }^{1,2}$ In this article, the authors raise awareness of $S$. suis infection in the Middle East and explore all the

common associations with S. suis by reporting the first case in the United Arab Emirates with bacteraemia, colon cancer, and no prior swine or pork contact.

\section{CASE PRESENTATION}

A 62-year-old Pakistani male presented with dizziness, nausea, and burning in micturition for 1 month prior to presentation. The patient also reported a $4 \mathrm{~kg}$ weight loss over a 3-month period. There was no history of fever. Past medical history was significant for diabetes and heart 
failure with reduced ejection fraction (40\%), as well as one previous history of extendedspectrum $\beta$-lactamase Escherichia coli cystitis 2 years ago. Social history was absent of direct animal contact and pork handling or eating. However, the patient reported eating meat from restaurants for 1 month prior to the onset of his symptoms. There was no recent travel history; the last time he left the country was over 1 year ago.

Upon initial presentation, his vitals were blood pressure $117 / 77 \mathrm{mmHg}$, pulse 78 beats per minute, temperature $37.2{ }^{\circ} \mathrm{C}$, respiratory rate 18 breaths per minute, and saturation of oxygen 95\% on room air. His physical examination did not reveal any abnormalities.

Laboratory investigation showed a white blood cell count of 13.4 per $\mathrm{mm}^{3}$, with $78 \%$ neutrophils and a haemoglobin count of $9.7 \mathrm{~g} / \mathrm{dL}$. The serum C-reactive protein (112.6 $\mathrm{mg} / \mathrm{dL}$ ) and procalcitonin (0.17; low risk for progression to severe sepsis) levels were elevated. Urinalysis showed positive nitrates with leukocyte esterase +3 and white blood cell count 10-15 per highpower field. Liver function tests showed alkaline phosphatase of 164 unit/L, alanine transaminase 16 unit/L, aspartate aminotransferase 28 unit/L, V-glutamyl transferase 123 unit/L, bilirubin $0.7 \mathrm{mg} / \mathrm{dL}$, albumin of $2.7 \mathrm{~g} / \mathrm{dL}$, and globulin of $3.9 \mathrm{~g} / \mathrm{dL}$.

As the patient was vitally stable with no signs of pyelonephritis, he was discharged on oral cefuroxime for 7 days after cultures from urine and blood were obtained. Three days later, the patient was called back to the hospital as his urine culture was growing extended-spectrum $\beta$-lactamase positive $E$. coli and his blood culture revealed the presence of gram-positive cocci in chains.

At presentation, the patient stated a partial improvement in the severity of his symptoms with the persistence of nausea. His vitals were blood pressure 133/83 $\mathrm{mmHg}$, pulse 85 beats per minute, temperature $36.6^{\circ} \mathrm{C}$, respiratory rate 16 breaths per minute, and saturation of oxygen $100 \%$ on room air. He was admitted and started on a daily dose of intravenous ertapenem $1 \mathrm{~g}$.

The patient's culture result was identified as $S$. suis 2 days after admission by means of matrixassisted laser desorption/ionisation time-of- flight (MALDI-TOF) through the automated mass spectrometry microbial identification VITEK system (BioMérieux, Marcy-l'Etoile, France). S. suis sensitivity was specifically requested for ertapenem as the patient was already prescribed it, and it was found to be sensitive. For his presenting anaemia with a mean corpuscular volume of 83, a blood film and iron studies were performed. The results were consistent with chronic anaemia.

A transthoracic echocardiogram was performed to exclude infective endocarditis and showed an ejection fraction of $35 \%$ with no vegetation.

Repeated blood cultures were negative, reducing further the likelihood of infective endocarditis. A renal ultrasound was performed to rule out any predisposing underlying urinary pathology to his recurrent infections. The ultrasound showed a normal urinary tract but revealed the presence of multiple heterogeneous liver masses with abnormal vascularity on the Doppler study (Figure 1). The serology for hepatitis B, C, and HIV was negative. Triphasic liver CT showed a malignant neoplastic process involving the sigmoid colon with metastatic liver lesions (Figure 2), as well as mesenteric and portocaval lymphadenopathy.

Colonoscopy was later performed, which showed a fungating mass with superficial ulcerations at the sigmoid colon (Figure 3). Biopsies obtained showed Grade 2 moderately-differentiated adenocarcinoma. No culture was obtained from the mass.

He was discharged in a stable condition after completing 10 days of ertapenem for his urinary tract infection and S. suis bacteraemia. A followup appointment with the oncology clinic was planned but the patient decided to travel to his home country for continuity of care.

\section{DISCUSSION}

S. suis is a gram-positive facultative anaerobe that is coccoid in shape and presents in chains or pairs. ${ }^{2}$ First identified in humans in $1968,{ }^{3}$ S. suis have since been reported to cause meningitis, sepsis, arthritis, and infective endocarditis. In a few reports, it has also caused endophthalmitis and osteomyelitis. ${ }^{4-7}$ 


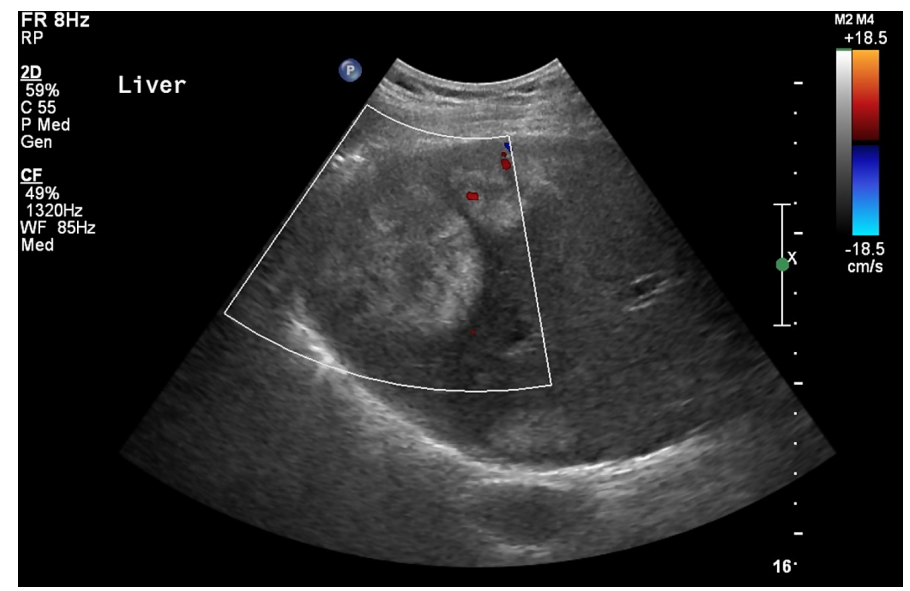

Figure 1: Ultrasound image of the liver showing multiple variable sizes of hyperechoic to hypoechoic heterogeneous masses in the liver showing no abnormal vascularity.

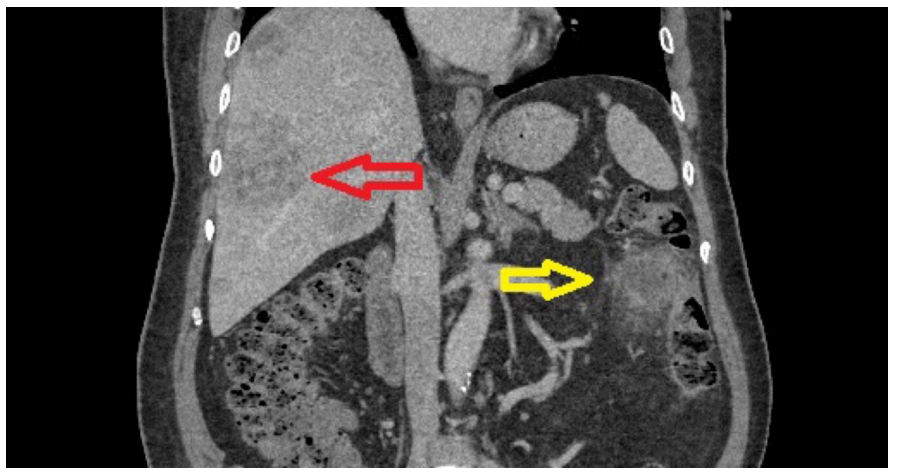

Figure 2: Coronal cut of abdominal CT in the portovenous phase showing the same sigmoid colon lesion (yellow arrow) as well as the liver metastasis (red arrow).

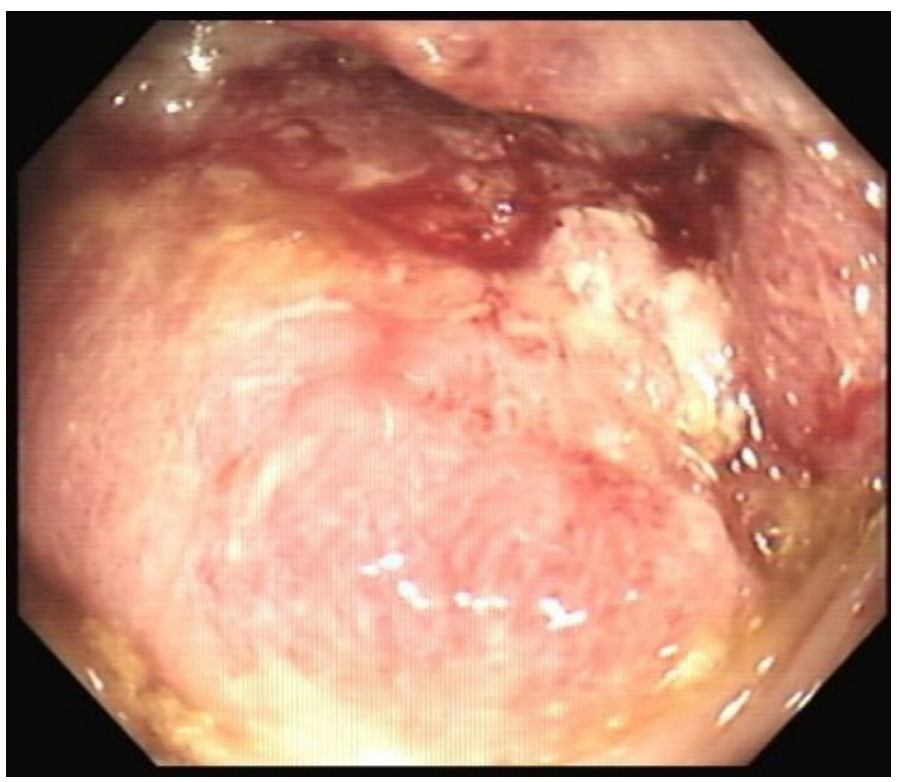

Figure 3: Direct visualisation by colonoscopy showing a fungating mass with ulceration. 
Human infection cases are reported worldwide, but mostly in Asian countries. ${ }^{4}$ Most of the reports are of sporadic cases, though outbreaks have been reported previously. In 2005, an outbreak in China resulted in 215 infected cases and 38 deaths. No person-to-person transmission was reported and all cases had direct contact with infected swine. ${ }^{8}$

Patients are generally healthy before infection with S. suis, although predisposing factors such as diabetes, alcoholism, and splenectomy have been reported.2,4 Male sex and occupations with direct swine or pork contact are considered risk factors. In a systemic review, swine-related occupations were present in $38.1 \%$ of cases and a history of eating high-risk food in $37.3 \%$ of cases. ${ }^{2}$ The risk is specifically high if pork is prepared in the presence of skin lesions. ${ }^{9}$

S. suis is a zoonotic organism that can be acquired through direct contact with swine or raw pork. ${ }^{2}$ It can also be acquired, albeit rarely, from other animals such as dogs or cats. ${ }^{10}$ Pork consumption is the usual method of transmission, especially high-risk foods such as fresh pig blood, or pig stomach, intestine, uterus, or tongue. This was confirmed in a case-control study that showed the presence of $S$. suis-specific DNA in a rectal swab of $6 / 100$ confirmed cases of $S$. suis meningitis, as compared to zero detection among the 1,522 healthy control cases. ${ }^{9}$ No stool culturing or swab was performed for the patient in this case; as a result, the method of transmission cannot be ascertained.

Not all cases, however, present with a history of contact with swine or other animals. One possibility of contracting $S$. suis infection can be through cross-contamination at places dealing with both meat and pork. A study in Thailand showed a high rate of contamination of $S$. suis in raw pork and edible pig organs sold in different areas." This is further supported by this patient's history of eating meat from restaurants around the time of symptom onset.

Another possibility is suggested by several reports of $S$. suis infection with no reported history of contact to pigs or pork. One case reported a 34-year-old male presenting with meningitis caused by $S$. suis with a negative history of pig or pork contact.12 Two other cases reported an association with cancer in patients presenting with $S$. suis infection and negative contact history: one was of metastatic oesophageal adenocarcinoma ${ }^{7}$ and the other was of a malignancy (location not described) found during workup. ${ }^{13}$

This case report is the second reported case of $S$. suis infection associated with colon cancer. The previously reported case was of infective endocarditis with bacteraemia caused by S. suis and an iron deficiency anaemia. Colonoscopy was performed as part of the anaemia workup, which revealed colon cancer. ${ }^{14}$ This case, however, had a positive animal contact with pigs as part of his occupation.

Based on these data, it can be concluded that $S$. suis is an occupational zoonotic infectious disease. However, in patients with no suggestive history of such high-risk contact, either directly or through cross-contamination of raw pork and meat, ruling out causes for an immunocompromised statelike malignancy is strongly advised. ${ }^{714}$

This conclusion, however, remains theoretical and is limited by several factors. First, despite the presence of $S$. suis bacteraemia with colon cancer in this case, this does not prove causation as it is unclear if the gut was the primary route of entry for the bacteraemia. Secondly, even with a negative contact history, ascertaining a true lack of exposure to pigs or pork is very difficult and, therefore, remains possible.

To the best of the authors' knowledge, this is the first report of $S$. suis infection in the United Arab Emirates and the Middle East region, and the second reported case with colon cancer association worldwide. This case should remind clinicians of the possibility of associated cancer in patients presenting with $S$. suis infection and no clear history of animal or pork contact. It should also come as a suggestion of the existence of colon cancer association with Streptococcus species other than bovis. ${ }^{14,15}$

\section{LEARNING POINTS/TAKE HOME MESSAGES}

> Patients presenting with S. suis infection may not have a positive contact history with pig or pork. 
> In patients with no obvious animal or pork contact history, a diagnosis of S. suis infection may raise the suspicion of underlying malignancy.

> Streptococcus spp. other than bovis can be associated with colon cancer.

\section{Ethics}

Informed consent was obtained from the patient and no ethical approval was required from the institute.

\section{References}

1. Rayanakorn A et al. Risk factors for Streptococcus suis infection: a systematic review and meta-analysis. Sci Rep. 2018;8(1):13358.

2. Huong VT et al. Epidemiology, clinical manifestations, and outcomes of Streptococcus suis infection in humans. Emerg Infect Dis. 2014;20(7):1105-14.

3. Wertheim HF et al. Streptococcus suis: an emerging human pathogen. Clin Infect Dis. 2009;48(5):617-25.

4. Dutkiewicz J et al. Streptococcus suis: a reemerging pathogen associated with occupational exposure to pigs or pork products. Part I epidemiology. Ann Agric Environ Med. 2017;24(4):683-95.

5. Sena Esteves S et al. Pig's ear: Streptococcus suis meningitis and its associated inner ear implications. ID Cases. 2017;10:55-7.
6. Mohapatra D et al. Chronic osteomyelitis due to Streptococcus Suis: first case report from India. J Glob Infect Dis. 2015;7(2):92-3.

7. Gomez-Zorrilla S et al. Streptococcus suis infection and malignancy in man, Spain. Emerg Infect Dis. 2014;20(6):1067-8

8. $\mathrm{Yu} \mathrm{H}$ et al. Human Streptococcus suis outbreak, Sichuan, China. Emerg Infect Dis. 2006;12(6):914-20.

9. Nghia HD et al. Risk factors of Streptococcus suis infection in Vietnam. A case-control study. PLoS One. 2011;6(3):e17604.

10. Salasia SI et al. Serotypes and putative virulence markers of Streptococcus suis isolates from cats and dogs. Res Vet Sci. 1994;57(2):259-61.
11. Boonyong $\mathrm{N}$ et al. Contamination of Streptococcus suis in pork and edible pig organs in central Thailand. Vet World. 2019;12(1):165-9.

12. Hidalgo A et al. Meningitis due to Streptococcus suis with no contact with pigs or porcine products. J Infect. 2007;55(5):478.

13. Manzin A et al. Streptococcus suis meningitis without history of animal contact, Italy. Emerg Infect Dis. 2008;14(12):1946-8.

14. Voutsadakis IA. Streptococcus suis endocarditis and colon carcinoma: a case report. Clin Colorectal Cancer. 2006;6(3):226-8.

15. Abdulamir AS et al. The association of Streptococcus bovis/gallolyticus with colorectal tumors: the nature and the underlying mechanisms of its etiological role. J Exp Clin Cancer Res. 2011;30:11. 